\title{
EXTREMAL BEHAVIOR OF ARCHIMEDEAN COPULAS
}

\author{
MARTIN LARSSON, ${ }^{*}$ Cornell University \\ JOHANNA NEŠLEHOVÁ,** McGill University
}

\begin{abstract}
We show how the extremal behavior of $d$-variate Archimedean copulas can be deduced from their stochastic representation as the survival dependence structure of an $\ell_{1}$ symmetric distribution (see McNeil and Nešlehová (2009)). We show that the extremal behavior of the radial part of the representation is determined by its Williamson $d$-transform. This leads in turn to simple proofs and extensions of recent results characterizing the domain of attraction of Archimedean copulas, their upper and lower tail-dependence indices, as well as their associated threshold copulas. We outline some of the practical implications of their results for the construction of Archimedean models with specific tail behavior and give counterexamples of Archimedean copulas whose coefficient of lower tail dependence does not exist.

Keywords: Archimedean copula; domain of attraction; extreme value copula; $\ell_{1}$-norm symmetric distribution; regular variation; simplex distribution; tail dependence; threshold copula; Williamson transform
\end{abstract}

2010 Mathematics Subject Classification: Primary 60G70

Secondary 60F05; 62E20

\section{Introduction}

Archimedean copulas form an important class of dependence models which enjoy considerable popularity in a number of fields, including biostatistics [9], [28], actuarial science, and quantitative finance [16], [26], [31, Chapter 10]. This is mainly due to their connection with frailty models and to the fact that they have an analytically tractable form, viz.

$$
C\left(u_{1}, \ldots, u_{d}\right)=\psi\left\{\psi^{-1}\left(u_{1}\right)+\cdots+\psi^{-1}\left(u_{d}\right)\right\}, \quad\left(u_{1}, \ldots, u_{d}\right) \in[0,1]^{d},
$$

where $\psi$ is a $d$-monotone function on $(0, \infty)$ such that $\psi(0)=1, \psi(x) \rightarrow 0$ as $x \rightarrow \infty$, and where, by convention, $\psi^{-1}(0)=\inf \{x \in[0, \infty): \psi(x)=0\}$; see [23], [24], and [32, Chapter 5]. Here, $d$-monotone means that $\psi$ has $d-2$ derivatives satisfying $(-1)^{k} \psi^{(k)} \geq 0$ on $(0, \infty)$ for $1 \leq k \leq d-2$, and that $(-1)^{d-2} \psi^{(d-2)}$ is nonincreasing and convex.

In this paper we focus on Archimedean copulas from an extreme value perspective. This issue has already received much attention in the literature; see [1], [5]-[7], [20], [21], and, in particular, [8]. However, the extremal behavior of these copulas has hitherto been characterized by the limiting behavior of either $\psi$ or its inverse at the endpoints of their domain. Though analytically convenient, these conditions provide only little probabilistic insight. It is also rather

Received 13 July 2009; revision received 23 August 2010.

* Postal address: Department of Operations Research and Information Engineering, Cornell University, 206 Rhodes Hall, Ithaca, NY 14853, USA.

** Postal address: Department of Mathematics and Statistics, McGill University, 805, rue Sherbrooke Ouest, Montréal (Québec), H3A 2K6, Canada. Email address: johanna@math.mcgill.ca 
difficult to construct new Archimedean generators $\psi$ that meet these conditions, or, in other words, new Archimedean copula models that exhibit specific tail behavior.

The idea pursued here is to characterize extremal behavior of Archimedean copulas using the stochastic representation recently developed in [24]. As shown by these authors, any $d$-dimensional Archimedean copula is the survival copula of a random vector $\boldsymbol{X}=\left(X_{1}, \ldots, X_{d}\right)$ following an $\ell_{1}$-norm symmetric distribution, i.e. $X \stackrel{\mathrm{D}}{=} R \boldsymbol{S}_{d}$, where $R$ is a random variable on $\mathbb{R}_{+}=[0, \infty)$ with $\operatorname{Pr}(R=0)=0$ and $S_{d}$ is an independent random vector which is uniformly distributed on the simplex

$$
\varsigma_{d}=\left\{\left(x_{1}, \ldots, x_{d}\right) \in \mathbb{R}_{+}^{d}: x_{1}+\cdots+x_{d}=1\right\} \subset \mathbb{R}_{+}^{d} .
$$

In addition, the Archimedean generator $\psi$ is the Williamson $d$-transform of the distribution function $F$ of the radial part $R$, i.e.

$$
\psi(x)=\mathfrak{W}_{d} F(x)=\int_{x}^{\infty}\left(1-\frac{x}{r}\right)^{d-1} \mathrm{~d} F(r), \quad x \in \mathbb{R}_{+} .
$$

In particular, $\psi$ is the survival function of each component of $\boldsymbol{X}$, i.e.

$$
\psi(x)=\operatorname{Pr}\left(X_{1}>x\right)=\cdots=\operatorname{Pr}\left(X_{d}>x\right)
$$

for all $x \in \mathbb{R}_{+}$. The distribution function $F$ may be retrieved from $\psi$ by means of the inverse Williamson $d$-transform, which is explicit. For any $x \in \mathbb{R}_{+}$, we have

$$
F(x)=\mathfrak{W}_{d}^{-1} \psi(x)=1-\sum_{k=0}^{d-2} \frac{(-1)^{k} x^{k} \psi^{(k)}(x)}{k !}-\frac{(-1)^{d-1} x^{d-1} \psi_{+}^{(d-1)}(x)}{(d-1) !},
$$

where $\psi_{+}^{(d-1)}$ is the right-hand derivative of the convex function $\psi^{(d-2)}$.

The connection between Archimedean copulas and $\ell_{1}$-norm symmetric distributions provides a systematic way of generating the full range of $d$-variate Archimedean copulas by specifying the distribution of the radial part $R$; see [25]. Although promising, this new modeling approach requires a better understanding of the relationship between the properties of the radial distribution and the dependence properties of the corresponding Archimedean copula. The present paper contributes to these investigations by showing that tail properties of an Archimedean copula are in fact characterized by extremal properties of its radial distribution.

It is first established in Section 2 that extremal behavior of the radial part can be conveniently characterized by analytical properties of its Williamson $d$-transform. The results presented there lead precisely to the conditions on $\psi$ or its inverse that are known to characterize the extremal behavior of the corresponding Archimedean copula. This fact sheds new light on many wellknown results pertaining to this class of copulas, which may thus be reproved and complemented easily. In Section 3 we discuss domain of attraction results, in Section 4 we focus on threshold copulas, and, finally, in Section 5 we consider coefficients of tail dependence. The latter section also includes three examples outlining practical implications for constructing new Archimedean models with targeted tail behavior. The first example introduces a new family of copulas whose upper and lower tail-dependence coefficients are flexibly governed by separate parameters and can attain all possible values; the two remaining examples are devoted to the construction of Archimedean copulas whose lower tail-dependence coefficient does not exist. Technical proofs from Section 2 are relegated to Appendices A and B. 


\section{Extremal behavior of the radial part}

Let $R_{1}, \ldots, R_{n}$ be independent copies of a random variable $R$ with distribution function $F$, and set

$$
M_{n}=\max \left(R_{1}, \ldots, R_{n}\right) .
$$

The variable $R$ is said to be in the maximum domain of attraction of an extreme value distribution $H$, denoted either $F \in \mathcal{M}(H)$ or $R \in \mathcal{M}(H)$, if and only if there exist sequences $\left(a_{n}\right)$ and $\left(b_{n}\right)$ of reals with $b_{n}>0$ such that

$$
\lim _{n \rightarrow \infty} \operatorname{Pr}\left(\frac{M_{n}-a_{n}}{b_{n}} \leq x\right)=H(x)
$$

holds for all $x \in \mathbb{R}$. Here, $H$ is either the Fréchet $\left(\Phi_{\alpha}\right)$, the Gumbel $(\Lambda)$, or the Weibull $\left(\Psi_{\alpha}\right)$ distribution with parameter $\alpha>0$. Conditions in terms of $F$ or $F^{-1}$ ensuring that $F \in \mathcal{M}(H)$ are standard; see, e.g. [12, Chapter 1.2], [14, Chapter 3.3], and [29, Chapter 1]. As is well known, the concept of regular variation is key in this context. A measurable function $f: \mathbb{R}_{+} \rightarrow \mathbb{R}_{+}$is called regularly varying with index $\rho \in \overline{\mathbb{R}}$ if, for any $x>0$,

$$
\lim _{t \rightarrow \infty} \frac{f(t x)}{f(x)}=x^{\rho}
$$

The class of regularly varying functions with index $\rho$ is denoted by $\mathcal{R}_{\rho}$.

Extensions of the concept of regular variation also play an important role. For a measurable function $a: \mathbb{R} \rightarrow(0, \infty)$, let $\Delta_{a}$ denote the class of all nonincreasing and right-continuous functions $f: \mathbb{R} \rightarrow[0, \infty)$ that satisfy

$$
\lim _{x \uparrow x_{f}} \frac{f\{x+a(x) t\}}{f(x)}=\mathrm{e}^{-t}, \quad t \in \mathbb{R},
$$

where $x_{f} \leq \infty$ is the upper endpoint of $f$, i.e. $x_{f}=\inf \{x: f(x)=0\}$. The function $a$ is referred to as the auxiliary function of $f$. To simplify the notation, write $f \in \Delta$ if $f \in \Delta_{a}$ for some $a$. Finally, a nondecreasing function $g$ is called $\Gamma$-varying if $1 / g \in \Delta$, whereas a nonincreasing and nonnegative function $h$ is called $\Pi$-varying if its left-continuous inverse $h^{\leftarrow}$ is $\Gamma$-varying. For more details on these classes of function, see [3, Chapter 3] and [29, Chapter 0.4.3]. Their significance originates from the fact that a distribution function $F$ lies in $\mathcal{M}(\Lambda)$ if and only if $\bar{F} \in \Delta$; see Proposition 0.10 of [29].

In order to study the tail behavior of Archimedean copulas, extremal behavior of the radial part needs to be characterized by its Williamson $d$-transform. This is done in Theorem 1 below, whose proof may be found in Appendix A.

Theorem 1. Let $R$ be a strictly positive random variable with distribution function $F$, upper endpoint $x_{F}=\sup \{x \in \mathbb{R}: F(x)<1\}$, and Williamsond-transform $\psi$ for some integer $d \geq 2$. Then the following statements hold for any $\alpha>0$.

(a) $R \in \mathcal{M}\left(\Phi_{\alpha}\right)$ if and only if $\psi \in \mathcal{R}_{-\alpha}$.

(b) $R \in \mathcal{M}\left(\Psi_{\alpha}\right)$ if and only if $\psi\left(x_{F}-x^{-1}\right) \in \mathcal{R}_{-\alpha-d+1}$.

(c) $R \in \mathcal{M}(\Lambda)$ if and only if $\psi \in \Delta$.

Note that, by (1) and (2), the upper endpoint $x_{F}$ of $F$ is precisely $\psi^{-1}(0)$. 
Remark 1. A side result that emerges from the proof of Theorem 1(a) is that $\psi$ is in fact the survival function of $R Y$, where $Y$ is a $\operatorname{Beta}(1, d-1)$ random variable independent of $R$. Statements (a)-(c) of Theorem 1 may thus be rephrased as follows: $R \in \mathcal{M}\left(\Phi_{\alpha}\right)$ if and only if $R Y \in \mathcal{M}\left(\Phi_{\alpha}\right) ; R \in \mathcal{M}\left(\Psi_{\alpha}\right)$ if and only if $R Y \in \mathcal{M}\left(\Psi_{\alpha+d-1}\right)$; and $R \in \mathcal{M}(\Lambda)$ if and only if $R Y \in \mathcal{M}(\Lambda)$.

It may further be seen from the proof of Theorem 1 (c) that, when $R \in \mathcal{M}(\Lambda)$,

$$
a_{1}(x)=\frac{\int_{x}^{x_{F}} \bar{F}(t) \mathrm{d} t}{\bar{F}(x)}, \quad a_{2}(x)=\frac{\int_{x}^{x_{F}} \psi(t) \mathrm{d} t}{\psi(x)}, \quad x \in(0, \infty),
$$

are possible auxiliary functions for both $\bar{F}$ and $\psi$. That is, $\bar{F}$ and $\psi$ are elements of $\Delta_{a_{i}}$ for $i=1,2$. The arguments in Appendix A also show that $R \in \mathcal{M}\left(\Psi_{\alpha}\right)$ if and only if $(-1)^{d-1} \psi_{+}^{(d-1)}\left(x_{F}-x^{-1}\right) \in \mathcal{R}_{-\alpha}$.

In addition to Theorem 1, it is useful to characterize the behavior of $R$ at 0 in terms of its Williamson $d$-transform. A suitable way to proceed is to consider the extremal behavior of $1 / R$. This is the purpose of the following result, proven in Appendix B.

Theorem 2. Let $R$ be a strictly positive random variable with Williamson d-transform $\psi$. Then the following statements hold.

(a) $1 / R \in \mathcal{M}\left(\Phi_{\alpha}\right)$ for $\alpha \in(0,1)$ if and only if $1-\psi\left(x^{-1}\right) \in \mathcal{R}_{-\alpha}$.

(b) $1-\psi\left(x^{-1}\right) \in \mathcal{R}_{-1}$ if either

(i) $1 / R \in \mathcal{M}\left(\Phi_{\alpha}\right)$ for $\alpha \in[1, \infty)$;

(ii) $1 / R \in \mathcal{M}(\Lambda)$; or

(iii) $1 / R \in \mathcal{M}\left(\Psi_{\alpha}\right)$ for $\alpha>0$.

Remark 2. In view of Theorem 2 , we may wonder what happens if $1-\psi\left(x^{-1}\right) \in \mathcal{R}_{-\alpha}$ for some $\alpha>1$. Because $\psi$ is $d$-monotone, however, this cannot occur. For,

$$
\alpha>1 \Longrightarrow \lim _{x \downarrow 0} \frac{1-\psi(x / 2)}{1-\psi(x)}=2^{-\alpha}<2^{-1} .
$$

In turn, this would imply the existence of an $x_{0} \in(0, \infty)$ such that $\psi(x / 2)>\{\psi(0)+\psi(x)\} / 2$ whenever $x \in\left(0, x_{0}\right)$, which would contradict the convexity of $\psi$. Similarly, in the context of Theorem 1(b), $\psi\left(x_{F}-x^{-1}\right) \in \mathcal{R}_{-\alpha}$ for $0<\alpha<d-1$ cannot occur either; see Proposition 4.9 of [22].

In some cases, it is easier to work with the inverse generator $\varphi=\psi^{-1}$ rather than $\psi$. The following result shows that extremal properties of $R$ and $1 / R$ may be characterized just as well in terms of $\varphi$.

Proposition 1. Let $\psi$ be a d-monotone Archimedean generator for some $d \geq 2$. Set $\varphi=\psi^{-1}$ and $x_{F}=\psi^{-1}(0)$. Then the following statements hold for any $\alpha>0$.

(a) $\psi \in \mathcal{R}_{-\alpha}$ if and only if $\varphi\left(x^{-1}\right) \in \mathcal{R}_{1 / \alpha}$.

(b) $\psi\left(x_{F}-x^{-1}\right) \in \mathcal{R}_{-\alpha-d+1}$ if and only if $\left\{x_{F}-\varphi\left(x^{-1}\right)\right\} \in \mathcal{R}_{-1 /(\alpha+d-1)}$.

(c) $\psi \in \Delta$ if and only if $\varphi\left(x^{-1}\right)$ is $\Pi$-varying.

(d) $1-\psi\left(x^{-1}\right) \in \mathcal{R}_{-\alpha}$ for $\alpha \in(0,1]$ if and only if $\varphi\left(1-x^{-1}\right) \in \mathcal{R}_{-1 / \alpha}$. 
Proof. To show (a), first note that, if $\psi \in \mathcal{R}_{-\alpha}, 1 / \psi$ is invertible and $1 / \psi \in \mathcal{R}_{\alpha}$. Thus, Theorem 1.5 .12 of [3] implies that $(1 / \psi)^{-1} \in \mathcal{R}_{1 / \alpha}$. However, for any $x \in(0, \infty),(1 / \psi)^{-1}(x)$ is precisely $\varphi(1 / x)$. The converse may be shown by reverting the argument.

The proofs of parts (b) and (d) are analogous to that of (a). Part (c) is a direct consequence of the definition of $\Pi$-variation, since $\varphi\left(x^{-1}\right)=(1 / \psi)^{-1}(x)$.

\section{Extremal behavior of Archimedean copulas}

Recall that a $d$-dimensional extreme value copula $C_{0}$ may be expressed as

$$
C_{0}\left(u_{1}, \ldots, u_{d}\right)=\exp \left[\log \left(u_{1} \cdots u_{d}\right) A\left\{\frac{\log \left(u_{1}\right)}{\log \left(u_{1} \cdots u_{d}\right)}, \ldots, \frac{\log \left(u_{d-1}\right)}{\log \left(u_{1} \cdots u_{d}\right)}\right\}\right],
$$

where the function $A$ is the so-called Pickands dependence function; see [15], [26, p. 312], and [33]. That is, $A:\left\{x \in[0,1]^{d-1}: x_{1}+\cdots+x_{d-1} \leq 1\right\} \rightarrow[0, \infty)$ is a function of the form

$$
A\left(x_{1}, \ldots, x_{d-1}\right)=\int_{\S_{d}} \max \left\{w_{1} x_{1}, \ldots, w_{d-1} x_{d-1}, w_{d}\left(1-\sum_{j=1}^{d-1} x_{j}\right)\right\} \mathrm{d} \mu\left(w_{1}, \ldots, w_{d}\right),
$$

where $\mu$ is a measure on $\wp_{d}$ satisfying $\int_{\delta_{d}} w_{j} \mathrm{~d} \mu(\boldsymbol{w})=1$ for all $1 \leq j \leq d$.

A $d$-dimensional copula $C$ is said to be in the maximum domain of attraction of an extreme value copula $C_{0}$, denoted $C \in \mathcal{M}\left(C_{0}\right)$, whenever

$$
\lim _{n \rightarrow \infty} C^{n}\left(u_{1}^{1 / n}, \ldots, u_{d}^{1 / n}\right)=C_{0}\left(u_{1}, \ldots, u_{d}\right)
$$

holds for any $\left(u_{1}, \ldots, u_{d}\right)$ in $[0,1]^{d}$. The purpose of this section is to establish conditions under which $C \in \mathcal{M}\left(C_{0}\right)$ holds and to identify the limits $C_{0}$ in the case when $C$ is a $d$-dimensional Archimedean copula or its survival counterpart. Here, a survival copula $\bar{C}$ of a copula $C$ is the distribution function of the vector $1-\boldsymbol{U}$, where $\boldsymbol{U}$ is distributed as $C$. These issues are addressed in turn in Sections 3.1 and 3.2.

\subsection{Extremal behavior of $C$}

Assume that $C$ is a $d$-dimensional Archimedean copula with generator $\psi$. Recall that $C$ is then the survival copula of an $\ell_{1}$-norm symmetric random vector $\boldsymbol{X}=\left(X_{1}, \ldots, X_{d}\right)$ with radial part $R$ whose Williamson $d$-transform is $\psi$.

Now consider $n$ independent replicates $\boldsymbol{X}_{i}=\left(X_{i 1}, \ldots, X_{i d}\right)$ of $\boldsymbol{X}, 1 \leq i \leq n$, and define $\boldsymbol{W}_{n}=\left(W_{n 1}, \ldots, W_{n d}\right)$ to be the vector of the componentwise minima, i.e.

$$
W_{n j}=\min \left(X_{1 j}, \ldots, X_{n j}\right), \quad 1 \leq j \leq d .
$$

As shown in [24], the survival function of $\boldsymbol{X}$ is given by

$$
\operatorname{Pr}\left(X_{1}>x_{1}, \ldots, X_{d}>x_{d}\right)=\psi\left(x_{1}+\cdots+x_{d}\right)=\psi(\|\boldsymbol{x}\|)
$$

for any $\boldsymbol{x}=\left(x_{1}, \ldots, x_{d}\right) \in \mathbb{R}_{+}^{d}$. Consequently, the survival function of $\boldsymbol{W}_{n}$ is

$$
\operatorname{Pr}\left(W_{n 1}>x_{1}, \ldots, W_{n d}>x_{d}\right)=\psi^{n}(\|\boldsymbol{x}\|), \quad \boldsymbol{x} \in \mathbb{R}_{+}^{d} .
$$

As the latter is a mere function of $\|\boldsymbol{x}\|, \boldsymbol{W}_{n}$ is again $\ell_{1}$-norm symmetric; see Proposition 3.3 of [24]. This has the following implication, which can be traced back to [5] and [17]. 
Proposition 2. Let $\psi$ be a d-monotone generator for some $d \geq 2$ such that $1-\psi\left(x^{-1}\right) \in \mathcal{R}_{-\alpha}$ for $\alpha \in(0,1]$. Then $C \in \mathcal{M}\left(C_{1 / \alpha}^{\mathrm{Gu}}\right)$, where $C_{\theta}^{\mathrm{Gu}}$ is the Gumbel copula with parameter $\theta \geq 1$, i.e. an Archimedean copula with generator $\psi_{\theta}^{\mathrm{Gu}}(x)=\exp \left[-x^{1 / \theta}\right]$, or, equivalently, an extreme value copula with Pickands dependence function $A_{\theta}^{\mathrm{Gu}}\left(x_{1}, \ldots, x_{d-1}\right)=\left\{x_{1}^{\theta}+\cdots+x_{d-1}^{\theta}+\right.$ $\left.\left(1-x_{1}-\cdots-x_{d-1}\right)^{\theta}\right\}^{1 / \theta}$.

Proof. In order to fit into the framework of the classical multivariate extreme value theory, consider the sequence $-\boldsymbol{X}_{i}, 1 \leq i \leq n$. It is immediate that $-\boldsymbol{W}_{n}$ is then the vector of the corresponding componentwise maxima. Furthermore, the margins of $-\boldsymbol{X}$ share the same distribution function $G(x)=\psi(-x), x \in(-\infty, 0]$. As $1-\psi\left(x^{-1}\right) \in \mathcal{R}_{-\alpha}$ by assumption, $G \in \mathcal{M}\left(\Psi_{\alpha}\right)$; see [14, Chapter 3.3.2] and [29, Chapter 1.3]. Thus, by (3) and Proposition 1.13 of [29], there exists a sequence $\left(b_{n}\right)$ of reals with $b_{n}>0$ such that

$$
\lim _{n \rightarrow \infty} \operatorname{Pr}\left(-\frac{W_{n 1}}{b_{n}} \leq x\right)=\lim _{n \rightarrow \infty} \psi^{n}\left(-b_{n} x\right)=\Psi_{\alpha}(x)=\exp \left[-(-x)^{\alpha}\right]
$$

whenever $x<0$. This implies that $\psi^{n}\left(-b_{n}\|\boldsymbol{x}\|\right)$ tends to $\psi_{1 / \alpha}^{\mathrm{Gu}}(-\|\boldsymbol{x}\|)$ as $n \rightarrow \infty$ for every $\boldsymbol{x} \in(-\infty, 0)^{d}$. Thus, by (5), $-\boldsymbol{W}_{\boldsymbol{n}} / b_{n}$ converges weakly to $-\boldsymbol{W}$, where $\boldsymbol{W}$ is an $\ell_{1}$-norm symmetric random vector with survival function $\psi_{1 / \alpha}^{\mathrm{Gu}}(-\|\boldsymbol{x}\|), \boldsymbol{x} \in \mathbb{R}_{+}^{d}$, and survival copula $C_{1 / \alpha}^{\mathrm{Gu}}$. By Theorem 7.48 of [26] we then have $C \in \mathcal{M}\left(C_{1 / \alpha}^{\mathrm{Gu}}\right)$.

Remark 3. In view of Theorem 2, the conditions of Proposition 2 are met if the radial part of $C$ satisfies either $1 / R \in \mathcal{M}(\Lambda), 1 / R \in \mathcal{M}\left(\Phi_{\alpha}\right)$, or $1 / R \in \mathcal{M}\left(\Psi_{\alpha}\right)$ for some $\alpha>0$. As $\operatorname{Pr}(1 / R>x)=\operatorname{Pr}(-R>-1 / x)$, this is equivalent to $-R \in \mathcal{M}\left(\Psi_{\alpha}\right)$ or $-R \in \mathcal{M}(\Lambda)$. Furthermore, it is of interest to note that if $1 / R \in \mathcal{M}\left(\Phi_{\alpha}\right)$ for some $\alpha \geq 1,1 / R \in \mathcal{M}(\Lambda)$, or $1 / R \in \mathcal{M}\left(\Psi_{\alpha}\right)$ for $\alpha>0$, then Theorem 2 implies that $1-\psi\left(x^{-1}\right) \in \mathcal{R}_{-1}$. Thus, the Archimedean copula with generator $\psi$ is in the maximum domain of attraction of the Gumbel copula with parameter $\theta=1$, which is the independence copula $\Pi\left(u_{1}, \ldots, u_{d}\right)=u_{1} \cdots u_{d}$.

The fact that the Gumbel family appears in the limit is not surprising in view of the following observation. Assume that $C$ is extreme value, which implies that

$$
\psi^{n}\left\{\psi^{-1}\left(u_{1}^{1 / n}\right)+\cdots+\psi^{-1}\left(u_{d}^{1 / n}\right)\right\}=\psi\left\{\psi^{-1}\left(u_{1}\right)+\cdots+\psi^{-1}\left(u_{d}\right)\right\}
$$

holds for every $u_{1}, \ldots, u_{d} \in[0,1]$ and $n \in \mathbb{N}$. Hence, the $\ell_{1}$-norm symmetric vectors $\boldsymbol{W}_{n}$ and $\boldsymbol{X}$ share the same survival copula for every $n \in \mathbb{N}$. By Proposition 2.14 of [22], this is possible if and only if there exist positive constants $b_{n}>0$ such that

$$
\psi(x)=\psi^{n}\left(b_{n} x\right)
$$

for any $x \geq 0$ and $n \in \mathbb{N}$. However, this means that the distribution function $G(x)=$ $\psi(-x), x \leq 0$, is max-stable by the Fisher-Tippett theorem; see, e.g. [14, Theorem 3.2.3]. Because its upper endpoint is finite, $G$ has to be the Weibull distribution $\Psi_{\alpha}(x)=\exp \left[-(-x)^{\alpha}\right]$. In addition, as argued in Section 2, its parameter must satisfy $\alpha \leq 1$. This implies that $\psi(x)=\exp \left[-x^{\alpha}\right]$, where the right-hand side is the Gumbel generator $\psi_{1 / \alpha}^{\mathrm{Gu}}$. In summary, this gives the following result, first obtained in [17].

Corollary 1. The Gumbel copulas $C_{\theta}^{\mathrm{Gu}}$ with parameter $\theta \geq 1$ are the only copulas that are both Archimedean and extreme value. 


\subsection{Extremal behavior of $\overline{\boldsymbol{C}}$}

Assume, as in Section 3.1, that $C$ is an Archimedean copula with generator $\psi$ and radial part $R$. Let $\boldsymbol{X}=R \boldsymbol{S}_{d}$ be an $\ell_{1}$-norm symmetric random vector whose survival copula is $C$. Given $n$ independent replicates $\boldsymbol{X}_{1}, \ldots, \boldsymbol{X}_{n}$ of $\boldsymbol{X}$, define $\boldsymbol{M}_{n}=\left(M_{n 1}, \ldots, M_{n d}\right)$ as the vector of the componentwise maxima, viz.

$$
M_{n j}=\max \left(X_{1 j}, \ldots, X_{n j}\right), \quad 1 \leq j \leq d .
$$

It is not difficult to see that, unlike the vector $\boldsymbol{W}_{n}$ of componentwise minima, $\boldsymbol{M}_{n}$ is no longer $\ell_{1}$-norm symmetric. Its limiting behavior can nonetheless be established using multivariate regular variation if $R$ is regularly varying. This leads to the following result, previously stated in [5] for the bivariate case.

Proposition 3. Let $C$ be an Archimedean copula with radial part $R \in \mathcal{M}\left(\Phi_{\alpha}\right)$ for some $\alpha>0$. Then $\bar{C} \in \mathcal{M}\left(C_{1 / \alpha}^{\mathrm{Ga}}\right)$, where $C_{\theta}^{\mathrm{Ga}}$ is the Galambos copula with parameter $\theta>0$, i.e. an extreme value copula with Pickands dependence function

$$
A_{\theta}^{\mathrm{Ga}}\left(x_{1}, \ldots, x_{d-1}\right)=\frac{1}{c_{\theta d}} \int_{\S_{d}} \max \left\{x_{1} w_{1}^{1 / \theta}, \ldots, x_{d-1} w_{d-1}^{1 / \theta},\left(1-\sum_{j=1}^{d-1} x_{j}\right) w_{d}^{1 / \theta}\right\} \mathrm{d} \sigma(\boldsymbol{w}),
$$

where $\sigma$ is the uniform distribution on $\varsigma_{d}, c_{\theta d}=(d-1) \mathrm{B}(1 / \theta+1, d-1)$, and $\mathrm{B}(\cdot, \cdot)$ denotes the beta function.

Proof. First note that $\|X\|=R$ is independent of $X /\|X\| \stackrel{\mathrm{D}}{=} S_{d}$. Consequently, by Proposition 1.11 of [29] there exists a sequence $\left(a_{n}\right)$ of reals $a_{n}>0$ such that, for any Borel set $A \subseteq \delta_{d}$ and $r>0$,

$$
\begin{aligned}
\lim _{n \rightarrow \infty} n \operatorname{Pr}\left(\|\boldsymbol{X}\|>a_{n} r, \frac{\boldsymbol{X}}{\|\boldsymbol{X}\|} \in A\right) & =\lim _{n \rightarrow \infty} n \operatorname{Pr}\left(R>a_{n} r\right) \operatorname{Pr}\left(\boldsymbol{S}_{d} \in A\right) \\
& =r^{-\alpha} \operatorname{Pr}\left(\boldsymbol{S}_{d} \in A\right) .
\end{aligned}
$$

In particular, therefore, Corollary 5.18 of [29] and Proposition 6.4 of [30] imply that $X$ is in the maximum domain of attraction of the multivariate extreme value distribution $H$ of the form

$$
H\left(x_{1}, \ldots, x_{d}\right)=\exp \left[-c \int_{\S_{d}} \max \left(\frac{w_{1}^{\alpha}}{x_{1}^{\alpha}}, \ldots, \frac{w_{d}^{\alpha}}{x_{d}^{\alpha}}\right) \mathrm{d} \sigma(\boldsymbol{w})\right] .
$$

Here, the sequence $\left(a_{n}\right)$ can be chosen in a way that $c=1$.

Next, observe that the univariate margins of $H$ are given by

$$
H_{j}\left(x_{j}\right)=\exp \left[-x_{j}^{-\alpha} \int_{\vartheta_{d}} w_{j}^{\alpha} \mathrm{d} \sigma(\boldsymbol{w})\right]=\exp \left[-(d-1) \mathrm{B}(\alpha+1, d-1) x_{j}^{-\alpha}\right]
$$

for every $1 \leq j \leq d$. Noting that $(d-1) \mathrm{B}(\alpha+1, d-1)=c_{(1 / \alpha) d}$, it is straightforward to verify that the copula of $H$ is indeed the Galambos copula $C_{1 / \alpha}^{\mathrm{Ga}}$. Consequently, Theorem 7.48 of [26] implies that $\bar{C} \in \mathcal{M}\left(C_{1 / \alpha}^{\mathrm{Ga}}\right)$, as claimed.

Example 1 justifies attaching the name of Galambos to the family of multivariate extreme value copulas $C_{\theta}^{\mathrm{Ga}}$, which also appears in [2]. 
Example 1. In the bivariate case, $c_{\theta 2}=1 /(1 / \theta+1)$. Furthermore, for any $x \in[0,1]$,

$$
\begin{aligned}
A_{\theta}^{\mathrm{Ga}}(x) & =\left(\frac{1}{\theta}+1\right) \int_{\S_{2}} \max \left\{w_{1}^{1 / \theta} x, w_{2}^{1 / \theta}(1-x)\right\} \mathrm{d} \sigma\left(w_{1}, w_{2}\right) \\
& =\left(\frac{1}{\theta}+1\right) \int_{0}^{1} \max \left\{w^{1 / \theta} x,(1-w)^{1 / \theta}(1-x)\right\} \mathrm{d} w \\
& =\left(\frac{1}{\theta}+1\right)(1-x) \int_{0}^{K}(1-w)^{1 / \theta} \mathrm{d} w+\left(\frac{1}{\theta}+1\right) x \int_{K}^{1} w^{1 / \theta} \mathrm{d} w
\end{aligned}
$$

where $K=(1-x)^{\theta} /\left\{x^{\theta}+(1-x)^{\theta}\right\}$. A straightforward calculation yields

$$
A_{\theta}^{\mathrm{Ga}}(x)=1-\left\{x^{-\theta}+(1-x)^{-\theta}\right\}^{-1 / \theta}, \quad x \in[0,1],
$$

which is the Pickands dependence function of the bivariate Galambos copula; see, e.g. Example 7.47 of [26].

If $R$ is either in the Gumbel or Weibull domain of attraction, the following holds.

Proposition 4. Assume that $C$ is an Archimedean copula with generator $\psi$ whose radial part satisfies either $R \in \mathcal{M}(\Lambda)$ or $R \in \mathcal{M}\left(\Psi_{\alpha}\right)$ for some $\alpha>0$. Then $\bar{C} \in \mathcal{M}(\Pi)$, where $\Pi$ is the independence copula.

Proof. Let $\boldsymbol{X}$ be the $\ell_{1}$-norm symmetric random vector whose survival copula is $C$. First consider the case where $\psi^{-1}(0)<\infty$. Then, clearly,

$$
\lim _{t \rightarrow x_{F}} \frac{\operatorname{Pr}\left(X_{i}>t, X_{j}>t\right)}{\psi(t)}=\lim _{t \rightarrow x_{F}} \frac{\psi(2 t)}{\psi(t)}=0, \quad 1 \leq i<j \leq d .
$$

This property remains valid even if $x_{F}=\infty$ and $R \in \mathcal{M}(\Lambda)$ because then $\psi \in \Delta$ by Theorem 1(c) and, consequently, $\psi$ is rapidly varying. Since all the margins of $\boldsymbol{X}$ are identically distributed, Proposition 5.27 of [29] implies that $\boldsymbol{X}$ is in the maximum domain of attraction of either $\Lambda\left(x_{1}\right) \cdots \Lambda\left(x_{d}\right)$ or $\Psi_{\alpha}\left(x_{1}\right) \cdots \Psi_{\alpha}\left(x_{d}\right)$ depending on whether $R \in \mathcal{M}(\Lambda)$ or $R \in \mathcal{M}\left(\Psi_{\alpha}\right)$. In any case, $\bar{C} \in \mathcal{M}(\Pi)$ by Theorem 7.48 of [26].

\section{Threshold copulas}

The purpose of this section is to examine the limiting threshold copulas of an Archimedean copula $C$. Sections 4.1 and 4.2 consider lower and upper threshold copulas, respectively.

\subsection{Limiting lower threshold copulas}

Let $\boldsymbol{U}$ be a random vector distributed as a $d$-dimensional Archimedean copula $C$ with generator $\psi$, and let $\boldsymbol{X}$ be an $\ell_{1}$-norm symmetric random vector with radial part $R$ whose survival copula is $C$. Given $v \in[0,1]^{d}$ with $C(v)>0$, the lower threshold copula $C_{\boldsymbol{v}}^{\ell}$ is defined as the copula of the conditional distribution function

$$
\operatorname{Pr}\left(U_{1} \leq u_{1}, \ldots, U_{d} \leq u_{d} \mid U_{1} \leq v_{1}, \ldots, U_{d} \leq v_{d}\right), \quad u_{i} \in\left[0, v_{i}\right], 1 \leq i \leq d .
$$

If $\psi^{-1}(0)=\infty$, the limiting lower threshold (LLT) copula of $C$ is given as the limit of $C_{v}^{\ell}$ as $v \rightarrow 0$, provided it exists. If $\psi$ is nonstrict, i.e. $\psi^{-1}(0)<\infty$, the so-called zero set $L(0)=\left\{\boldsymbol{u} \in(0,1)^{d}: C(\boldsymbol{u})=0\right\}$ is nonempty. In this case, the LLT copula of $C$ is 
the limit (if it exists) of $C_{v}^{\ell}$ as $v$ approaches some $v_{0}$ on the boundary of the zero set, i.e. $\boldsymbol{v}_{0} \in\left\{\boldsymbol{u} \in(0,1)^{d}: \psi^{-1}\left(u_{1}\right)+\cdots+\psi^{-1}\left(u_{d}\right)=\psi^{-1}(0)\right\}$. The LLT copula could in principle depend on $\boldsymbol{v}_{0}$ or the rate of convergence of $\boldsymbol{v}$ to $\boldsymbol{v}_{0}$; however, as will soon become clear, this is of no concern if $C$ is Archimedean.

As $\psi^{-1}$ is strictly decreasing on $(0,1], C_{v}^{\ell}$ is the survival copula of $H_{z}$, where

$$
\begin{aligned}
\bar{H}_{z}(\boldsymbol{x}) & =\operatorname{Pr}\left(X_{1} \geq x_{1}+z_{1}, \ldots, X_{d} \geq x_{d}+z_{d} \mid X_{1} \geq z_{1}, \ldots, X_{d} \geq z_{d}\right) \\
& =\frac{\psi(\|\boldsymbol{x}\|+\|z\|)}{\psi(\|z\|)}
\end{aligned}
$$

for $\boldsymbol{z}=\left(\psi^{-1}\left(v_{1}\right), \ldots, \psi^{-1}\left(v_{d}\right)\right)$ and $\boldsymbol{x} \in \mathbb{R}_{+}^{d}$. Because $\bar{H}_{z}(\boldsymbol{x})$ is a function of $\|\boldsymbol{x}\|$ alone, $\bar{H}_{z}$ is again $\ell_{1}$-norm symmetric. This leads to the following result, which extends [21].

Proposition 5. Let $C$ be a d-dimensional Archimedean copula with generator $\psi$ and radial part $R$. Furthermore, let $C_{\theta}^{\mathrm{Cl}}$ be the Clayton copula, i.e. an Archimedean copula with generator $\psi_{\theta}^{\mathrm{Cl}}(x)=(1+\theta x)_{+}^{-1 / \theta}, \theta \geq-1 /(d-1)$. Then the following statements hold.

(a) If $R \in \mathcal{M}\left(\Phi_{\alpha}\right)$ for $\alpha>0$, the LLT copula of $C$ is $C_{1 / \alpha}^{\mathrm{Cl}}$.

(b) If $R \in \mathcal{M}(\Lambda)$, the LLT copula of $C$ is $\Pi$.

(c) If $R \in \mathcal{M}\left(\Psi_{\alpha}\right)$ for $\alpha>0$, the LLT copula of $C$ is $C_{-1 /(\alpha+d-1)}^{\mathrm{Cl}}$.

Proof. Let $\bar{G}_{\theta}(x)$ denote the survival function of the generalized Pareto distribution [14, Chapter 3.4]. Observe that, for $x>0, \bar{G}_{\theta}(x)=\mathrm{e}^{-x}$ if $\theta=0$ and $\bar{G}_{\theta}(x)=\psi_{\theta}^{\mathrm{Cl}}(x)$ otherwise. Next, note that $\bar{H}_{z}(\boldsymbol{x})$ is the excess distribution of $\psi$ at $\|\boldsymbol{x}\|$ with threshold $\|z\|$. By the PickandsBalkema-de Haan theorem [14, Theorem 3.4.5] and Theorem 1, there exists a positive and measurable function $\beta$ such that

$$
\lim _{\|z\| \uparrow \psi^{-1}(0)} \bar{H}_{z}\{x \beta(\|z\|)\}=\bar{G}_{\theta}(\|x\|), \quad x \in \mathbb{R}_{+}^{d},
$$

where $\theta=1 / \alpha$ in case (a), $\theta=0$ in case (b), and $\theta=-1 / \alpha$ in case (c). Because copulas are invariant with respect to scaling, the survival copula of $\bar{H}_{z}\{x \beta(\|z\|)\}$ is again $C_{v}^{\ell}$. Since the margins of both $\bar{H}_{z}\{\boldsymbol{x} \beta(\|z\|)\}$ and $\bar{G}_{\theta}(\|\boldsymbol{x}\|)$ are continuous, this implies that, as $\|\boldsymbol{z}\| \uparrow \psi^{-1}(0)$, $C_{v}^{\ell}$ converges to the survival copula of $\bar{G}_{\theta}(\|x\|)$, which is precisely the Clayton copula $C_{\theta}^{\mathrm{Cl}}$, as claimed. In addition, if $\boldsymbol{v}$ tends to an arbitrary $\boldsymbol{v}_{0} \in\left\{\boldsymbol{u} \in(0,1)^{d}: \psi^{-1}\left(u_{1}\right)+\cdots+\psi^{-1}\left(u_{d}\right)=\right.$ $\left.\psi^{-1}(0)\right\},\|z\| \uparrow \psi^{-1}(0)$.

\subsection{Limiting upper threshold copulas}

Limiting upper threshold copulas of Archimedean copulas turn out to be less tractable. In this section we discuss only partial results under two additional assumptions. Firstly, let the radial part $R$ of an Archimedean copula $C$ be such that

$$
\inf \{x \in \mathbb{R}: \operatorname{Pr}(R \leq x)>0\}=0 .
$$

This condition guarantees that if $\boldsymbol{U}$ is a random vector distributed as $C$,

$$
\operatorname{Pr}\left(U_{1}>v_{1}, \ldots, U_{d}>v_{d}\right)>0, \quad\left(v_{1}, \ldots, v_{d}\right) \in(0,1)^{d} .
$$

Secondly, only thresholds of the form $v=(v, \ldots, v)$ for some $v \in(0,1)$ will be considered. For any such $v$, the upper threshold copula $C_{v}^{u}$ of $C$ may be defined as the copula of the conditional distribution with survival function

$$
\operatorname{Pr}\left(U_{1}>u_{1}, \ldots, U_{d}>u_{d} \mid U_{1}>v, \ldots, U_{d}>v\right), \quad u_{i} \in[v, 1], 1 \leq i \leq d .
$$


The limiting upper threshold (LUT) copula of $C$ is then the limit of $C_{v}^{u}$ as $v \rightarrow 1$, provided it exists.

Using the same notation as in Section 4.1 and setting $z=\psi^{-1}(v)$, it is easily seen that $C_{v}^{u}$ is the survival copula of the conditional distribution

$$
K_{z}\left(x_{1}, \ldots, x_{d}\right)=\operatorname{Pr}\left(X_{1} \leq x_{1}, \ldots, X_{d} \leq x_{d} \mid X_{1} \leq z, \ldots, X_{d} \leq z\right),
$$

where $x_{i} \in[0, z], 1 \leq i \leq d$. Because the support of $K_{z}$ is $[0, z]^{d}, K_{z}$ cannot be $\ell_{1}$-norm symmetric. Nonetheless, its limit as $z \rightarrow 0$ can be computed under suitable conditions on the radial part. This leads to the following result, which is in agreement with [8].

Proposition 6. Let $C$ be an Archimedean copula with generator $\psi$, and assume that its radial part satisfies $1 / R \in \mathcal{M}\left(\Phi_{\alpha}\right)$ for $\alpha \in(0,1)$. Then the LUT copula of $C$ is the survival copula of the distribution function defined for all $x_{1}, \ldots, x_{d} \in[0,1]$ by

$$
K\left(x_{1}, \ldots, x_{d}\right)=\frac{\sum_{k=1}^{d}(-1)^{k-1} \sum_{1 \leq i_{1}<\cdots<i_{k} \leq d}\left(x_{i_{1}}+\cdots+x_{i_{d}}\right)^{\alpha}}{\sum_{k=1}^{d}\left(\begin{array}{l}
d \\
k
\end{array}\right)(-1)^{k-1} k^{\alpha}} .
$$

Proof. First note that, if $1 / R \in \mathcal{M}\left(\Phi_{\alpha}\right)$ holds, condition (6) is automatically fulfilled. Using the inclusion-exclusion principle, we can further see that

$$
\begin{aligned}
\operatorname{Pr}\left(X_{1} \leq x_{1}, \ldots, X_{d} \leq x_{d}\right) & =1+\sum_{k=1}^{d}(-1)^{k} \sum_{1 \leq i_{1}<\cdots<i_{k} \leq d} \psi\left(x_{i_{1}}+\cdots+x_{i_{d}}\right) \\
& =\sum_{k=1}^{d}(-1)^{k-1} \sum_{1 \leq i_{1}<\cdots<i_{k} \leq d}\left\{1-\psi\left(x_{i_{1}}+\cdots+x_{i_{d}}\right)\right\} .
\end{aligned}
$$

This means that, for $x_{i} \in(0,1), 1 \leq i \leq d$,

$$
\begin{aligned}
K_{z}\left(z x_{1}, \ldots, z x_{d}\right) & =\frac{\sum_{k=1}^{d}(-1)^{k-1} \sum_{1 \leq i_{1}<\cdots<i_{k} \leq d}\left[1-\psi\left\{z\left(x_{i_{1}}+\cdots+x_{i_{d}}\right)\right\}\right]}{\sum_{k=1}^{d}(-1)^{k-1}\left(\begin{array}{l}
d \\
k
\end{array}\right)\{1-\psi(k z)\}} \\
& =\frac{\sum_{k=1}^{d}(-1)^{k-1} \sum_{1 \leq i_{1}<\cdots<i_{k} \leq d}\left[1-\psi\left\{z\left(x_{i_{1}}+\cdots+x_{i_{d}}\right)\right\}\right] /\{1-\psi(z)\}}{\sum_{k=1}^{d}(-1)^{k-1}\left(\begin{array}{l}
d \\
k
\end{array}\right)\{1-\psi(k z)\} /\{1-\psi(z)\}} .
\end{aligned}
$$

Recalling that $1-\psi\left(x^{-1}\right) \in \mathcal{R}_{-\alpha}$ for $\alpha \in(0,1)$ by Theorem 2 , we conclude that

$$
\lim _{z \downarrow 0} K_{z}\left(z x_{1}, \ldots, z x_{d}\right)=K\left(x_{1}, \ldots, x_{d}\right), \quad x_{i} \in(0,1), 1 \leq i \leq d .
$$

Consequently, the survival copula of the distribution function $K_{z}\left(z x_{1}, \ldots, z x_{d}\right), x_{i} \in(0,1)$, $1 \leq i \leq d$, converges to the survival copula of $K$. Because copulas are invariant with respect to strictly monotone transformations of the margins, the former is $C_{v}^{u}$.

Although the margins of $K$ are identical, the inverse of their distribution function is not available in closed form. As a consequence, neither the copula nor the survival copula of $K$ is algebraically tractable. This happens already when $d=2$. Indeed,

$$
K\left(x_{1}, x_{2}\right)=\frac{x_{1}^{\alpha}+x_{2}^{\alpha}-\left(x_{1}+x_{2}\right)^{\alpha}}{2-2^{\alpha}}, \quad\left(x_{1}, x_{2}\right) \in[0,1]^{2},
$$

with identical margins equal to $\left\{1+x^{\alpha}-(1+x)^{\alpha}\right\} /\left(2-2^{\alpha}\right)$ for all $x \in[0,1]$. 


\section{Coefficients of lower and upper tail dependences}

For a bivariate copula $C$, coefficients of lower and upper tail dependences are defined in [19] by

$$
\lambda_{\mathrm{L}}=\lim _{q \rightarrow 0} \frac{C(q, q)}{q}, \quad \lambda_{\mathrm{U}}=\lim _{q \rightarrow 1} \frac{1-2 q+C(q, q)}{1-q},
$$

provided the limits exist. If $C$ is a $d$-dimensional Archimedean copula with generator $\psi$, all its bivariate margins are also Archimedean with the same generator. It is easy to check that their coefficients of upper and lower tail dependence are given by

$$
\lambda_{\mathrm{L}}=\left\{\begin{array}{ll}
\lim _{x \rightarrow \infty} \psi(2 x) / \psi(x) & \text { if } \psi^{-1}(0)=\infty, \\
0 & \text { otherwise, }
\end{array} \quad \lambda_{\mathrm{U}}=2-\lim _{x \rightarrow 0} \frac{1-\psi(2 x)}{1-\psi(x)}\right.
$$

These expressions show that $\lambda_{\mathrm{L}}$ and $\lambda_{\mathrm{U}}$ are related to the regular variation of $\psi$ at $\infty$ and $1-\psi$ at 0 , respectively. The next result, which follows directly from Theorems 1 and 2, links these coefficients with the extremal behavior of $R$.

Corollary 2. Let $C$ be a d-dimensional Archimedean copula with generator $\psi$ and radial part $R$. Then the following statements hold.

(a) If $R \in \mathcal{M}\left(\Phi_{\alpha}\right)$ then $\lambda_{\mathrm{L}}=2^{-\alpha}$.

(b) If $R \in \mathcal{M}\left(\Psi_{\alpha}\right)$ or $R \in \mathcal{M}(\Lambda)$, then $\lambda_{\mathrm{L}}=0$.

(c) If $1 / R \in \mathcal{M}\left(\Phi_{\alpha}\right)$ for $\alpha \in(0,1)$ then $\lambda_{\mathrm{U}}=2-2^{\alpha}$.

(d) If $1 / R \in \mathcal{M}\left(\Psi_{\alpha}\right), 1 / R \in \mathcal{M}(\Lambda)$, or $1 / R \in \mathcal{M}\left(\Phi_{\alpha}\right)$ for $\alpha \geq 1$, then $\lambda_{\mathrm{U}}=0$.

The same expressions for $\lambda_{\mathrm{L}}$ and $\lambda_{\mathrm{U}}$ were previously obtained in [8] under conditions on the inverse of $\psi$. However, Theorems 1 and 2 combined with Proposition 1 easily relate Corollary 2 to the results of Sections 3 and 4 of [8].

Remark 4. The statement in Corollary 2(a) can be compared to similar results on the taildependence coefficients of elliptical copulas. These dependence structures are copulas of elliptical distributions, whose stochastic representation is similar to the $\ell_{1}$-norm symmetric distributions. More specifically, a random vector $\boldsymbol{X}$ follows an elliptical distribution with mean $\mu$, positive definite covariance matrix $\boldsymbol{\Sigma}$, and generator $\phi$ if and only if $\boldsymbol{X} \stackrel{\mathrm{D}}{=} \mu+T \boldsymbol{A U}$, where $\boldsymbol{A}$ is a $d \times d$ matrix such that $\boldsymbol{A} \boldsymbol{A}^{\top}=\boldsymbol{\Sigma}$, and $T$ is a nonnegative random variable independent of a random vector $\boldsymbol{U}$, uniformly distributed on the unit hypersphere $\left\{\boldsymbol{x} \in \mathbb{R}^{d}: \boldsymbol{x}^{\top} \boldsymbol{x}=1\right\}$. Under suitable regularity conditions on $\boldsymbol{\Sigma}$, it was shown in [18] that, if $T \in \mathcal{M}\left(\Phi_{\alpha}\right)$, the coefficient of lower tail dependence of any bivariate margin $\left(X_{i}, X_{j}\right)$ of $\boldsymbol{X}$ is positive and depends only on $\alpha$ and $\rho_{i j}=\Sigma_{i j} / \sqrt{\Sigma_{i i} \Sigma_{j j}}$. Because elliptical copulas are radially symmetric, $\lambda_{\mathrm{U}}\left(X_{i}, X_{j}\right)=\lambda_{\mathrm{L}}\left(X_{i}, X_{j}\right)$, which is not the case for Archimedean copulas.

Commonly used families of Archimedean copulas have the property that either $\lambda_{L}=\lambda_{U}$ or that only one of the coefficients is nonzero. The following example shows how this fact, which is often restrictive in applications, may be overcome using Corollary 2.

Example 2. Consider a radial part $R$ whose distribution function is given by

$$
F_{\alpha, \kappa}(x)= \begin{cases}x^{\kappa} / 2 & \text { if } x \leq 1 \\ 1-x^{-\alpha} / 2 & \text { if } x>1\end{cases}
$$



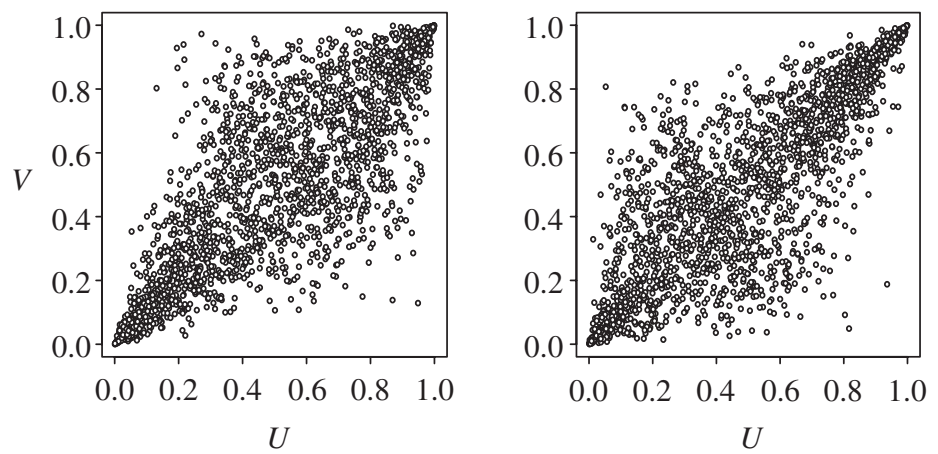

FIGURE 1: Samples from $C_{\alpha, \kappa}$ with $\alpha=\frac{1}{3}, \kappa=\frac{1}{2}$ (left) and $\alpha=\frac{2}{3}, \kappa=\frac{1}{4}(r i g h t)$.

with parameters $\kappa>0$ and $\alpha>0$. It is easy to see that $R \in \mathcal{M}\left(\Phi_{\alpha}\right)$ while $1 / R \in \mathcal{M}\left(\Phi_{\kappa}\right)$. As a consequence, the coefficients of tail dependence for a bivariate Archimedean copula $C_{\alpha, \kappa}$ with radial distribution $F_{\alpha, \kappa}$ are $\lambda_{\mathrm{L}}=2^{-\alpha}$ and $\lambda_{\mathrm{U}}=2-2^{\min (\kappa, 1)}$. This means that, for this family of copulas, all possible combinations of the values of $\lambda_{\mathrm{L}}$ and $\lambda_{\mathrm{U}}$ are attainable. Figure 1 shows samples of size 2000 from $C_{\alpha, \kappa}$ with parameters such that $\lambda_{\mathrm{L}} \approx 0.794$ and $\lambda_{\mathrm{U}} \approx 0.586$ (left panel) and $\lambda_{\mathrm{L}} \approx 0.629$ and $\lambda_{\mathrm{U}} \approx 0.811$ (right panel).

The coefficients of tail dependence exist for all commonly encountered families of Archimedean copulas (see, e.g. [27, Example 5.22] and Tables 1 and 2 of [8]). With the relatively strong $d$-monotonicity condition on $\psi$ in mind, it is tempting to conjecture that such is always the case. What follows next are two intricate counterexamples.

Example 3. Fix $d \geq 2$, and define a function

$$
\psi_{a}(x)=\frac{1+a \sin \{\log (1+x)\}}{1+x}, \quad x \in \mathbb{R}_{+},
$$

with parameter $a<1$. As shown next, $\psi_{a}$ is a $d$-monotone Archimedean generator for suitable choices of $a$. Clearly, $\psi_{a}(0)=1, \lim _{x \rightarrow \infty} \psi_{a}(x)=0$, and $\psi_{a}(x)>0$ for all $x \geq 0$. Furthermore, it is easily established by induction that, for any $k \geq 0$,

$$
\psi_{a}^{(k)}(x)=(-1)^{k} \frac{k !+a c_{k} \sin \left\{\varphi_{k}+\log (1+x)\right\}}{(1+x)^{k+1}}, \quad x \geq 0 .
$$

Here, $c_{k}$ and $\varphi_{k}$ are constants given recursively by $c_{0}=1, c_{k}=c_{k-1} \sqrt{k^{2}+1}$ and $\varphi_{0}=0$, $\varphi_{k}=\varphi_{k-1}+\arctan (1 / k)$. Because the sine function is bounded below by -1 , the condition $(-1)^{k} \psi_{a}^{(k)}(x) \geq 0$ holds for all $x>0$ whenever $0<a \leq k ! / c_{k}$. Since $c_{0}<c_{1}<\cdots<c_{d}$, choosing $a \in\left(0,1 / c_{d}\right)$ ensures that $\psi_{a}$ is a $d$-monotone generator.

To show that $\psi_{a}$ is not regularly varying at $\infty$, consider the sequence $x_{n}=\mathrm{e}^{n \pi}$ which diverges as $n \rightarrow \infty$. Then $\psi\left(c x_{n}\right) / \psi\left(x_{n}\right)=f\left(x_{n}\right)\left(1+x_{n}\right) /\left(1+c x_{n}\right)$, where

$$
\begin{aligned}
f\left(x_{n}\right) & =\frac{1+a \sin \left\{\log \left(1+c x_{n}\right)\right\}}{1+a \sin \left\{\log \left(1+x_{n}\right)\right\}} \\
& =\frac{1+a \sin \left\{n \pi+\log \left(\mathrm{e}^{-n \pi}+c\right)\right\}}{1+a \sin \left\{n \pi+\log \left(\mathrm{e}^{-n \pi}+1\right)\right\}} \\
& =\frac{1+a(-1)^{n} \sin \left\{\log \left(\mathrm{e}^{-n \pi}+c\right)\right\}}{1+a(-1)^{n} \sin \left\{\log \left(\mathrm{e}^{-n \pi}+1\right)\right\}} .
\end{aligned}
$$


It is easily seen that the limit of $f\left(x_{n}\right)$ as $n \rightarrow \infty$ does not exist. Indeed, $f\left(x_{2 n}\right) \rightarrow 1+$ $a \sin (\log c)$, while $f\left(x_{2 n+1}\right) \rightarrow 1-a \sin (\log c)$ as $n \rightarrow \infty$. In particular, therefore, the limit of $\psi\left(c x_{n}\right) / \psi\left(x_{n}\right)$ as $n \rightarrow \infty$ does not exist unless $\log c=m \pi$ for some integer $m \geq 1$. Consequently, $\psi_{a}$ for $a \in\left(0,1 / c_{d}\right)$ generates an Archimedean copula in dimension $d$ whose bivariate margins do not have a coefficient of lower tail dependence.

The next example shows that even the stronger requirement of complete monotonicity of $\psi$ is not enough to guarantee the existence of the coefficient of lower tail dependence.

Example 4. Define $f(x)=K \sum_{k=0}^{\infty} \mathbf{1}_{\left(2^{-2 k-1}, 2^{-2 k}\right]}(x)$, where the constant $K$ is chosen so that $\int_{0}^{\infty} f(x) \mathrm{d} x=1$, and set $F(x)=\int_{0}^{x} f(y) \mathrm{d} y$. Now let $\psi$ be the Laplace transform of $F$; by construction, $\psi$ is a completely monotone Archimedean generator. We readily compute that

$$
\psi(t)=\frac{K}{t} \sum_{k=0}^{\infty}\left(\exp \left[-t 2^{-2 k-1}\right]-\exp \left[-t 2^{-2 k}\right]\right)
$$

and, hence, for an arbitrary $c>0$,

$$
\frac{\psi(c t)}{\psi(t)}=\frac{1}{c} \frac{\sum_{k=0}^{\infty}\left(\exp \left[-c t 2^{-2 k-1}\right]-\exp \left[-c t 2^{-2 k}\right]\right)}{\sum_{k=0}^{\infty}\left(\exp \left[-t 2^{-2 k-1}\right]-\exp \left[-t 2^{-2 k}\right]\right)} .
$$

It turns out that, for $c=2$, the limit as $t \rightarrow \infty$ of the ratio $\psi(2 t) / \psi(t)$ does not exist, which implies that the bivariate Archimedean copula generated by $\psi$ does not have a coefficient of lower tail dependence. This fact is established below by showing that the limits of $\psi(2 \cdot) / \psi$ along the subsequences $t_{n}=2^{2 n}$ and $s_{n}=2^{2 n+1}$ differ.

First, the sum in the expression for $\psi\left(t_{n}\right)$ may be rewritten as

$$
\begin{aligned}
& \sum_{k=0}^{\infty}\left(\exp \left[-t_{n} 2^{-2 k-1}\right]-\exp \left[-t_{n} 2^{-2 k}\right]\right) \\
& \quad=\sum_{k=n}^{\infty}\left(\exp \left[-2^{2 n-2 k-1}\right]-\exp \left[-2^{2 n-2 k}\right]\right)+\sum_{k=0}^{n-1}\left(\exp \left[-2^{2 n-2 k-1}\right]-\exp \left[-2^{2 n-2 k}\right]\right) .
\end{aligned}
$$

The first sum on the right-hand side equals $\sum_{k=0}^{\infty}\left(\exp \left[-2^{-2 k-1}\right]-\exp \left[-2^{-2 k}\right]\right)$; setting $j=$ $n-k$ in the second sum results in $\sum_{j=1}^{n}\left(\exp \left[-2^{2 j-1}\right]-\exp \left[-2^{2 j}\right]\right)$. In particular, therefore,

$$
\lim _{n \rightarrow \infty} \sum_{k=0}^{\infty}\left(\exp \left[-t_{n} 2^{-2 k-1}\right]-\exp \left[-t_{n} 2^{-2 k}\right]\right)=\sum_{k=-\infty}^{\infty}\left(\exp \left[-2^{-2 k-1}\right]-\exp \left[-2^{-2 k}\right]\right) .
$$

It is easy to check that the sum on the right-hand side is finite and even absolutely convergent. Turning to the sum in the expression for $\psi\left(2 t_{n}\right)$, analogous calculations yield

$$
\lim _{n \rightarrow \infty} \sum_{k=0}^{\infty}\left(\exp \left[-2 t_{n} 2^{-2 k-1}\right]-\exp \left[-2 t_{n} 2^{-2 k}\right]\right)=\sum_{k=-\infty}^{\infty}\left(\exp \left[-2^{-(2 k+1)-1}\right]-\exp \left[-2^{-(2 k+1)}\right]\right)
$$

Because $s_{n}=2 t_{n}$ and $2 s_{n}=t_{n+1}$, these observations imply that

$$
\lim _{n \rightarrow \infty} \frac{\psi\left(2 t_{n}\right)}{\psi\left(t_{n}\right)}=\frac{1}{2} \frac{\sum_{k=-\infty}^{\infty}\left(\exp \left[-2^{-2 k-1}\right]-\exp \left[-2^{-2 k}\right]\right)}{\sum_{k=-\infty}^{\infty}\left(\exp \left[-2^{-(2 k+1)-1}\right]-\exp \left[-2^{-(2 k+1)}\right]\right)}
$$


while

$$
\lim _{n \rightarrow \infty} \frac{\psi\left(2 s_{n}\right)}{\psi\left(s_{n}\right)}=\frac{1}{2} \frac{\sum_{k=-\infty}^{\infty}\left(\exp \left[-2^{-(2 k+1)-1}\right]-\exp \left[-2^{-(2 k+1)}\right]\right)}{\sum_{k=-\infty}^{\infty}\left(\exp \left[-2^{-2 k-1}\right]-\exp \left[-2^{-2 k}\right]\right)} .
$$

The limits as $n \rightarrow \infty$ of $\psi\left(2 t_{n}\right) / \psi\left(t_{n}\right)$ and $\psi\left(2 s_{n}\right) / \psi\left(s_{n}\right)$ thus differ if and only if

$$
\sum_{k=-\infty}^{\infty}\left(\exp \left[-2^{-(2 k+1)-1}\right]-\exp \left[-2^{-(2 k+1)}\right]\right) \neq \sum_{k=-\infty}^{\infty}\left(\exp \left[-2^{-2 k-1}\right]-\exp \left[-2^{-2 k}\right]\right)
$$

To verify this claim, set $a_{\ell}=\exp \left[-2^{-\ell-1}\right]-\exp \left[-2^{-\ell}\right]$. Since $a_{\ell} \geq 0$ for any $\ell \in \mathbb{Z}$, we have

$$
\begin{aligned}
\sum_{k=-\infty}^{\infty} a_{2 k+1}-\sum_{k=-\infty}^{\infty} a_{2 k} & =\sum_{k=-2}^{5} a_{2 k+1}-\sum_{k=-2}^{6} a_{2 k}+\sum_{\substack{k \leq-3 \\
k \geq 6}} a_{2 k+1}-\sum_{\substack{k \leq-3 \\
k \geq 7}} a_{2 k} \\
& \geq \sum_{k=-2}^{5} a_{2 k+1}-\sum_{k=-2}^{6} a_{2 k}-\sum_{\substack{\ell \leq-5 \\
\ell \geq 13}} a_{\ell} \\
& =\sum_{k=-2}^{5} a_{2 k+1}-\sum_{k=-2}^{6} a_{2 k}-\left(1-\exp \left[-2^{-13}\right]-\exp \left[-2^{4}\right]\right),
\end{aligned}
$$

where the last equality follows from the telescopic property and the fact that $\sum_{\ell \in \mathbb{Z}} a_{\ell}=1$. The value of the lower bound was found to be strictly positive (greater than $10^{-4}$ ) by direct computation. This completes the counterexample.

The cases where $\lambda_{\mathrm{L}}=0$ and $\lambda_{\mathrm{U}}=0$ are referred to as asymptotic independence in the lower and upper tails, respectively; see Propositions 2 and 4. In [11], the authors introduced the socalled coefficients of tail dependence of the second kind, which provide additional information in such situations. The latter are given by

$$
\mu_{\mathrm{L}}=\lim _{q \rightarrow 0} \frac{2 \log q}{\log C(q, q)}-1, \quad \mu_{\mathrm{U}}=\lim _{q \rightarrow 1} \frac{2 \log (1-q)}{\log \{1-2 q+C(q, q)\}}-1 .
$$

As shown in [11] and [13], $\mu_{\mathrm{L}}=1$ whenever $\lambda_{\mathrm{L}}>0$ and $\mu_{\mathrm{L}}>-1$ whenever $\lambda_{\mathrm{L}}=0$, and similarly for $\mu_{\mathrm{U}}$. The following result illustrates that in the case of asymptotic independence, $\mu_{\mathrm{L}}$ may be computed under second-order conditions on the tail behavior of the radial part.

Proposition 7. Let $C$ be a bivariate Archimedean copula with differentiable generator $\psi$. Assume further that the radial part $R$ of $C$ is such that $R \in \mathcal{M}(\Lambda)$ and $x_{F}=\infty$. If the auxiliary function a of $R$ satisfies $a \in \mathcal{R}_{\beta}$ for some $\beta \leq 1$ then $\mu_{\mathrm{L}}=2^{\beta}-1$.

Proof. Substituting $t=\psi^{-1}(q)$ and using l'Hôpital's rule, we obtain

$$
\lim _{q \rightarrow 0} \frac{2 \log q}{\log C(q, q)}=\lim _{t \rightarrow \infty} \frac{2 \log \psi(t)}{\log \psi(2 t)}=\lim _{t \rightarrow \infty} \frac{\psi(2 t) / \psi^{\prime}(2 t)}{\psi(t) / \psi^{\prime}(t)} .
$$

From Theorem 1 and Remark $1, \psi \in \Delta$ with auxiliary function $a$. The result thus follows by regular variation of $a$ if $a$ and $-\psi / \psi^{\prime}$ are tail equivalent, i.e. $-a(x) \psi^{\prime}(x) / \psi(x) \rightarrow 1$ as $x \rightarrow \infty$. To show this, note that $\psi(x)=-\int_{x}^{\infty} \psi^{\prime}(t) \mathrm{d} t$, so that, by Lemma 1 in Appendix A, $-\psi^{\prime} \in \Delta_{a}$. As is well known (see, e.g. Proposition 1.9 of [29]), $-\int_{x}^{\infty} \psi^{\prime}(t) \mathrm{d} t /\left\{-\psi^{\prime}(x)\right\}=-\psi(x) / \psi^{\prime}(x)$ is a valid choice of auxiliary function for $-\psi^{\prime}$. Thus, $a$ and $-\psi / \psi^{\prime}$ must be tail equivalent. 
Remark 5. Note that Proposition 7 is in agreement with Section 3.3 of [8], where the same expression for $\mu_{\mathrm{L}}$ is derived under the assumption of regular variation of $-\psi / \psi^{\prime}$. The arguments in the proof of Proposition 7 show that the latter condition is equivalent to the requirement that the auxiliary function $a$ of $R$ satisfies $a \in \mathcal{R}_{\beta}$.

As already indicated in Theorem 5.1 of [8], the condition $\beta \leq 1$ in Proposition 7 is not restrictive, because we cannot have $a \in \mathcal{R}_{\beta}$ for $\beta>1$. Indeed, Theorem 1 implies that $\psi \in \Delta$. In particular, therefore, $\psi(x) / \exp \left\{-\int_{z_{0}}^{x} \mathrm{~d} t / a(t)\right\} \rightarrow 1$ as $x \rightarrow \infty$ for some $z_{0}>0$. If $\beta>1$, we would then have $\int_{0}^{\infty} \mathrm{d} t / a(t)<\infty$ by Karamata's theorem [3, Theorem 1.5.11], [29, Theorem 0.6] and, hence, $\lim _{x \rightarrow \infty} \psi(x)>0$, which is a contradiction.

Similar calculations are possible for the coefficient $\mu_{\mathrm{U}}$. To obtain nondegenerate results, we need to consider second-order regular variation of $1-\psi$, which goes beyond the scope of the present paper. For more results on this issue, see [8].

\section{Appendix A. Proof of Theorem 1}

To simplify the notation in the proof, let $\psi^{(d-1)}$ be the right-hand derivative of $\psi^{(d-2)}$. Furthermore, $f \sim g$ for two real functions $f$ and $g$ means that $f(x) / g(x) \rightarrow 1$ as $x \rightarrow \infty$.

Proof of $(a)$. Let $Y$ be a $\operatorname{Beta}(1, d-1)$ random variable which is independent of $R$, and observe that

$$
\psi(x)=\int_{0}^{\infty}\left(1-\frac{x}{r}\right)^{d-1} \mathrm{~d} F(r)=\int_{0}^{\infty} \bar{G}\left(\frac{x}{r}\right) \mathrm{d} F(r)=\operatorname{Pr}(R Y>x),
$$

where $G$ denotes the distribution function of $Y$. Since $\mathrm{E}\left(Y^{\alpha+\varepsilon}\right)<\infty$ for all $\varepsilon>0$, Breiman's theorem [4], [10] implies that $\psi(x)=\operatorname{Pr}(R Y>x) \in \mathcal{R}_{-\alpha}$.

Conversely, suppose that $\psi(x)=x^{-\alpha} L(x)$ for some slowly varying function $L$. As is shown below by induction, we have

$$
(-1)^{k} x^{k} \psi^{(k)}(x) \sim \prod_{j=0}^{k-1}(j+\alpha) \psi(x)
$$

for $k=0, \ldots, d-1$. The claim is trivial for $k=0$, and because $\psi$ is $d$-monotone,

$$
(-1)^{k} \psi^{(k)}(x)=\int_{x}^{\infty}(-1)^{k+1} \psi^{(k+1)}(y) \mathrm{d} y
$$

for $k=0, \ldots, d-2$. Now suppose that $(-1)^{k} \psi^{(k)}(x) \sim \prod_{j=0}^{k-1}(j+\alpha) x^{-k} \psi(x)$. In other words, $(-1)^{k} \psi^{(k)}(x) \sim c_{k} x^{-k-\alpha} L(x)$, where $c_{k}=\prod_{j=0}^{k-1}(j+\alpha)$ is a positive constant. Since $(-1)^{k+1} \psi^{(k+1)}$ is nonincreasing, the monotone density theorem [3, Theorem 1.7.2], [14, Theorem A 3.7] implies that $(-1)^{k+1} \psi^{(k+1)}(x) \sim c_{k}(k+\alpha) x^{-k-\alpha-1} L(x)$. Hence, $(-1)^{k+1} x^{k+1} \times$ $\psi^{(k+1)}(x) \sim \prod_{j=0}^{k}(j+\alpha) \psi(x)$, as claimed.

Now, use (7) and (2) for the inverse Williamson $d$-transform to see that

$$
\frac{1-F(x)}{\psi(x)}=\frac{1-\mathfrak{W}_{d}^{-1} \psi(x)}{\psi(x)}=\sum_{k=0}^{d-1} \frac{1}{k !} \frac{(-1)^{k} x^{k} \psi^{(k)}(x)}{\psi(x)} \rightarrow \sum_{k=0}^{d-1} \frac{1}{k !} \prod_{j=0}^{k-1}(j+\alpha)
$$

as $x \rightarrow \infty$. As $\alpha>0$, the limit is positive. This implies that $\bar{F} \in \mathcal{R}_{-\alpha}$. 
Proof of $(b)$. Assume that $R \in \mathcal{M}\left(\Psi_{\alpha}\right)$ for some $\alpha>0$. As is well known, this happens if and only if $\bar{F}\left(x_{F}-x^{-1}\right) \in \mathcal{R}_{-\alpha}$; see, e.g. [14, Theorem 3.3.12] and [29, Chapter 1.3]. Using the definition of the Williamson $d$-transform and dominated convergence, we easily obtain

$$
(-1)^{(d-1)} \psi^{(d-1)}(x)=(d-1) ! \int_{x}^{\infty} t^{-d+1} \mathrm{~d} F(t),
$$

which can be rewritten as follows by partial integration:

$$
(-1)^{d-1} \psi^{(d-1)}(x)=(d-1) !\left\{x^{-d+1} \bar{F}(x)-(d-1) \int_{x}^{\infty} t^{-d} \bar{F}(t) \mathrm{d} t\right\} .
$$

The change of variable $t=x_{F}-s^{-1}$ then leads to

$$
\begin{aligned}
(-1)^{d-1} \psi^{(d-1)}\left(x_{F}-x^{-1}\right)=(d-1) ! & \left\{\left(x_{F}-x^{-1}\right)^{-d+1} \bar{F}\left(x_{F}-x^{-1}\right)\right. \\
& \left.-(d-1) \int_{x}^{\infty} s^{-2}\left(x_{F}-s^{-1}\right)^{-d} \bar{F}\left(x_{F}-s^{-1}\right) \mathrm{d} s\right\} .
\end{aligned}
$$

Now, write $\bar{F}\left(x_{F}-x^{-1}\right)=x^{-\alpha} L(x)$ for some slowly varying function $L$. As $K(x)=$ $\left(x_{F}-x^{-1}\right)^{-d} L(x)$ is again slowly varying, Karamata's theorem [3, Theorem 1.5.11], [29, Theorem 0.6] yields

$$
\frac{\int_{x}^{\infty} s^{-\alpha-2} K(s) \mathrm{d} s}{x^{-\alpha-1} K(x)} \rightarrow \frac{1}{\alpha+1}
$$

as $x \rightarrow \infty$. In particular, therefore,

$$
\begin{aligned}
& \frac{(-1)^{d-1} \psi^{(d-1)}\left(x_{F}-x^{-1}\right)}{\bar{F}\left(x_{F}-x^{-1}\right)} \\
& \quad=(d-1) !\left\{\left(x_{F}-x^{-1}\right)^{-d+1}-\frac{(d-1)\left(x_{F}-x^{-1}\right)^{-d}}{x} \frac{\int_{x}^{\infty} s^{-\alpha-2} K(s) \mathrm{d} s}{x^{-\alpha-1} K(x)}\right\},
\end{aligned}
$$

which converges to $(d-1) ! x_{F}^{-d+1}$ as $x \rightarrow \infty$. Hence, $(-1)^{d-1} \psi^{(d-1)}\left(x_{F}-x^{-1}\right) \in \mathcal{R}_{-\alpha}$. Now because $\psi^{(k)}(x)=0$ whenever $x>x_{F}$, we have

$$
\begin{aligned}
(-1)^{k} \psi^{(k)}\left(x_{F}-x^{-1}\right) & =\int_{x_{F}-x^{-1}}^{x_{F}}(-1)^{k+1} \psi^{(k+1)}(t) \mathrm{d} t \\
& =\int_{x}^{\infty}(-1)^{k+1} s^{-2} \psi^{(k+1)}\left(x_{F}-s^{-1}\right) \mathrm{d} s
\end{aligned}
$$

for $k=0, \ldots, d-2$. Thus, successive applications of Karamata's theorem yield

$$
(-1)^{k} \psi^{(k)}\left(x_{F}-x^{-1}\right) \in \mathcal{R}_{-\alpha-(d-1)+k}
$$

for $k=0, \ldots, d-2$. In particular, this implies that $\psi\left(x_{F}-x^{-1}\right) \in \mathcal{R}_{-\alpha-(d-1)}$, as claimed.

For the converse, suppose that

$$
\psi\left(x_{F}-x^{-1}\right) \in \mathcal{R}_{-\alpha-(d-1)},
$$

i.e. $\psi\left(x_{F}-x^{-1}\right)=x^{-\alpha-(d-1)} L(x)$ for some slowly varying function $L$. Because $\alpha>0$ by 
assumption, $-\alpha-(d-1)+k<0$ for every $k=0, \ldots, d-1$. Invoking (10) and the monotone density theorem repeatedly, we obtain

$$
(-1)^{k} \psi^{(k)}\left(x_{F}-x^{-1}\right) \sim a_{k} x^{-\alpha-(d-1)+k} L(x) \quad \text { for } k=0, \ldots, d-1,
$$

where $a_{k}=\prod_{j=0}^{k-1}(\alpha+d-1-j)$ is a strictly positive constant. Now, the inversion formula (2) gives

$$
\frac{\bar{F}\left(x_{F}-x^{-1}\right)}{x^{-\alpha} L(x)}=\sum_{k=0}^{d-1} \frac{\left(x_{F}-x^{-1}\right)^{k} x^{k-(d-1)}(-1)^{k} \psi^{(k)}\left(x_{F}-x^{-1}\right)}{x^{-\alpha-(d-1)+k} L(x) k !}
$$

for all sufficiently large $x$. As $x \rightarrow \infty$, the right-hand side converges to $a_{d-1}\left(x_{F}\right)^{d-1} /(d-1)$ !. In other words, $F\left(x_{F}-x^{-1}\right) \in \mathcal{R}_{-\alpha}$ and the proof is complete.

The proof of Theorem 1 (c) requires several technical results related to $\Gamma$-varying functions, including modifications of Corollary 3.10.7 and Theorem 3.10.11 of [3].

Lemma 1. The following statements hold.

(a) If $f \in \Delta_{a}$ satisfies $f(x)=\int_{x}^{\infty} g(t) \mathrm{d} t$ for all $x \in \mathbb{R}$ and some nonincreasing and right-continuous function $g$, then $g \in \Delta_{a}$.

(b) Let $f \in \Delta_{a}$ and $g(x)=\int_{x}^{\infty} f(t) \mathrm{d} t$ be well defined for all $x \in \mathbb{R}$. Then $g \in \Delta_{a}$.

(c) Let $f \in \Delta_{a}, \rho \in \mathbb{R}$, and $g(x)=x^{\rho} f(x), x \in \mathbb{R}$. Then $g \in \Delta_{a}$.

(d) Let $g=f \pm f^{*}$, where $f, f^{*} \in \Delta_{a}$ with the same upper endpoint $x_{f}$ and such that $\lim _{x \uparrow x_{f}} f(x) / f^{*}(x)=\ell$ for some $\ell \in[0, \infty)$. Then $g \in \Delta_{a}$.

Proof. To show (a), first note that because $f$ is nonincreasing, $g$ is nonnegative with $x_{g}=x_{f}$. Now pick two reals $t<s$. Then, for every $x \in \mathbb{R}$,

$$
\frac{f\{x+a(x) t\}-f\{x+a(x) s\}}{f(x)}=\frac{1}{f(x)} \int_{x+a(x) t}^{x+a(x) s} g(v) \mathrm{d} v=\frac{a(x)}{f(x)} \int_{t}^{s} g\{x+a(x) u\} \mathrm{d} u .
$$

As $g$ is nonincreasing, this implies that

$$
\frac{f\{x+a(x) t\}-f\{x+a(x) s\}}{f(x)} \leq \frac{a(x) g\{x+a(x) t\}(s-t)}{f(x)} .
$$

Hence, recalling that $f \in \Delta_{a}$ by assumption,

$$
\liminf _{x \uparrow x_{f}} \frac{a(x) g\{x+a(x) t\}}{f(x)} \geq \lim _{x \uparrow x_{f}} \frac{f\{x+a(x) t\}-f\{x+a(x) s\}}{f(x)(s-t)}=\frac{\mathrm{e}^{-t}-\mathrm{e}^{-s}}{s-t} .
$$

Letting $s \rightarrow t$, this means that $\liminf _{x \uparrow x_{f}} a(x) g\{x+a(x) t\} / f(x) \geq \mathrm{e}^{-t}$. Similarly, we can argue that $\lim \sup _{x \uparrow x_{f}} a(x) g\{x+a(x) t\} / f(x) \leq \mathrm{e}^{-t}$. Consequently,

$$
\lim _{x \uparrow x_{f}} \frac{a(x) g\{x+a(x) t\}}{f(x)}=\mathrm{e}^{-t}, \quad t \in \mathbb{R} .
$$

The case $t=0$ yields $a(x) g(x) / f(x) \rightarrow 1$ as $x \rightarrow x_{f}$. Thus, $g\{x+a(x) t\} / g(x) \rightarrow \mathrm{e}^{-t}$ for every $t \in \mathbb{R}$, which implies that $g \in \Delta_{a}$, as claimed. 
Regarding (b), note that, if $f \in \Delta_{a}$, the auxiliary function $a$ satisfies

$$
\lim _{x \uparrow x_{f}} \frac{a(x)}{x}=0 ;
$$

see, e.g. Lemma 3.10.1 of [3]. Furthermore, according to Propositions 0.9 and 0.14 of [29], we have $f \in \Delta_{a^{*}}$ with the asymptotically equivalent auxiliary function

$$
a^{*}(x)=\int_{x}^{x_{f}} \frac{f(t) \mathrm{d} t}{f(x)}=\frac{g(x)}{f(x)}, \quad x \in \mathbb{R} .
$$

Now, by Proposition 3.10.6 of [3], $a^{*}$ is self-neglecting. This implies that

$$
\lim _{x \uparrow x_{f}} \frac{a^{*}\left\{x+t a^{*}(x)\right\}}{a^{*}(x)}=\lim _{x \uparrow x_{f}} \frac{g\left\{x+t a^{*}(x)\right\} f(x)}{f\left\{x+t a^{*}(x)\right\} g(x)}=1 .
$$

Consequently, $g$ satisfies (4) and, hence, $g \in \Delta_{a^{*}}$, which is the same as $g \in \Delta_{a}$.

Part (c) follows directly with (11) and the fact that $f$ satisfies (4). Indeed,

$$
\lim _{x \uparrow x_{f}} \frac{g\{x+a(x) t\}}{g(x)}=\lim _{x \uparrow x_{f}}\left(1+\frac{a(x)}{x} t\right)^{\rho} \frac{f\{x+a(x) t\}}{f(x)}=\mathrm{e}^{-t} .
$$

To show (d), write $\eta(x)=f(x) / f^{*}(x)$ and consider the case $g=f+f^{*}$. Now

$$
\frac{f\{x+a(x) t\}+f^{*}\{x+a(x) t\}}{f(x)+f^{*}(x)}=\frac{f\{x+a(x) t\} / f(x)+\eta(x) f^{*}\{x+a(x) t\} / f^{*}(x)}{1+\eta(x)} .
$$

Clearly, the right-hand side tends to $\mathrm{e}^{-t}$ as $x \rightarrow x_{f}$. The case $g=f-f^{*}$ is similar.

Proof of $(c)$. Suppose first that $R \in \mathcal{M}(\Lambda)$. As is well known, this is equivalent to $\bar{F} \in \Delta_{a}$ for some auxiliary function $a$. Because of (8) and Lemma 1(b), it is sufficient to show that $(-1)^{d-1} \psi^{(d-1)} \in \Delta_{a}$. To do so, recall (9) and observe that $(-1)^{d-1} \psi^{(d-1)}(x)=\gamma(x)-\delta(x)$ for $x \in(0, \infty)$, where

$$
\gamma(x)=(d-1) ! x^{-d+1} \bar{F}(x), \quad \delta(x)=(d-1) !(d-1) \int_{x}^{x_{F}} t^{-d} \bar{F}(t) \mathrm{d} t .
$$

Now by Lemma 1(c) and (b), $\gamma \in \Delta_{a}$ as well as $\delta \in \Delta_{a}$. However, because of Propositions 0.9 and 0.14 of [29], the function

$$
a^{*}(x)=\frac{\int_{x}^{x_{F}} t^{-d} \bar{F}(t) \mathrm{d} t}{x^{-d} \bar{F}(x)}, \quad x \in(0, \infty),
$$

is another auxiliary function for $\delta$. In particular, (11) holds for $a^{*}$ and, thus, $\delta(x) / \gamma(x)=$ $(d-1) a^{*}(x) / x$ tends to 0 as $x \rightarrow x_{F}$. Consequently, $\gamma-\delta$ satisfies (4) by Lemma 1(d). As $(-1)^{d-1} \psi^{(d-1)}$ is nonincreasing and right continuous, it is in the class $\Delta_{a}$.

To show the converse, recall the inversion formula (2), i.e.

$$
\bar{F}(x)=\sum_{k=0}^{d-1} \frac{(-1)^{k} x^{k} \psi^{(k)}(x)}{k !}, \quad x \in(0, \infty)
$$


and observe that each of the summands satisfies (4) by (8) and Lemma 1(a) and (c). Thus, $\bar{F} \in \Delta_{a}$ by Lemma $1(\mathrm{~d})$ provided that

$$
\xi_{k}(x)=\frac{(d-1) !(-1)^{k} x^{k} \psi^{(k)}(x)}{k !(-1)^{d-1} x^{d-1} \psi^{(d-1)}(x)}
$$

has a finite limit as $x \rightarrow x_{F}$ for every $k=0, \ldots, d-2$. Because $(-1)^{k} \psi^{(k)} \in \Delta_{a}$ by (8) and Lemma 1(a), the function $a_{k}^{*}$ given by

$$
a_{k}^{*}(x)=\frac{\int_{x}^{x_{F}}(-1)^{k} \psi^{(k)}(t) \mathrm{d} t}{(-1)^{k} \psi^{(k)}(x)}=\frac{(-1)^{k-1} \psi^{(k-1)}(x)}{(-1)^{k} \psi^{(k)}(x)}, \quad x \in(0, \infty),
$$

is a possible auxiliary function for $(-1)^{k} \psi^{(k)}$ as per Propositions 0.9 and 0.14 of [29] for all $k=1, \ldots, d-1$. Now write

$$
\begin{aligned}
\frac{k ! \xi_{k}(x)}{(d-1) !} & =\frac{(-1)^{k} x^{k} \psi^{(k)}(x)}{(-1)^{k+1} x^{k+1} \psi^{(k+1)}(x)} \frac{(-1)^{k+1} x^{k+1} \psi^{(k+1)}(x)}{(-1)^{k+2} x^{k+2} \psi^{(k+2)}(x)} \cdots \frac{(-1)^{d-2} x^{d-2} \psi^{(d-2)}(x)}{(-1)^{d-1} x^{d-1} \psi^{(d-1)}(x)} \\
& =\frac{a_{k+1}^{*}(x)}{x} \frac{a_{k+2}^{*}(x)}{x} \cdots \frac{a_{d-1}^{*}(x)}{x} .
\end{aligned}
$$

Because each $a_{k}^{*}$ satisfies (11), the last expression tends to 0 as $x \rightarrow x_{F}$. Thus, Lemma 1(d) implies that $\bar{F} \in \Delta_{a}$ as announced, and the proof is complete.

\section{Appendix B. Proof of Theorem 2}

Proof of $(a)$. Fix $\alpha \in(0,1)$, and assume that $1 / R \in \mathcal{M}\left(\Phi_{\alpha}\right)$. If $Y$ is a $\operatorname{Beta}(1, d-1)$ random variable which is independent of $R$, it is easy to see that

$$
1-\psi\left(\frac{1}{x}\right)=\operatorname{Pr}\left\{\frac{1}{R Y}>x\right\}, \quad x \in(0, \infty) .
$$

Furthermore, we can easily check that $\mathrm{E}\left(Y^{-\alpha-\varepsilon}\right)<\infty$ if $\varepsilon$ is sufficiently small to ensure that $\alpha+\varepsilon<1$. Thus, Breiman's theorem implies that $\operatorname{Pr}\{1 /(R Y)>x\}=1-\psi(1 / x) \in \mathcal{R}_{-\alpha}$, as claimed.

For the converse, suppose that $1-\psi(1 / x)=x^{-\alpha} L(x)$ for some slowly varying function $L$. Consequently, $1-\psi(x)$ is regularly varying at 0 with index $\alpha$ and because

$$
1-\psi(x)=-\int_{0}^{x} \psi^{\prime}(t) \mathrm{d} t
$$

the monotone density theorem implies that $-\psi^{\prime}(1 / x) \sim \alpha x^{1-\alpha} L(x)$. Given that (8) holds for $1 \leq k \leq d-2$, successive applications of the same theorem yield

$$
(-1)^{k} x^{-k} \psi^{(k)}\left(\frac{1}{x}\right) \sim b_{k} x^{-\alpha} L(x)
$$

for $k=1, \ldots, d-2$ as well as $(-1)^{d-1} x^{1-d} \psi_{-}^{(d-1)}(1 / x) \sim b_{d-1} x^{-\alpha} L(x)$. Here, $b_{k}=$ $\alpha \prod_{j=1}^{k-1}(j-\alpha)$ is a positive constant, $k=1, \ldots, d-1$. From (2),

$$
\begin{aligned}
\frac{\operatorname{Pr}(R<1 / x)}{x^{-\alpha} L(x)}= & \frac{1-\psi(1 / x)}{x^{-\alpha} L(x)}-\sum_{k=1}^{d-2} \frac{(-1)^{k} x^{-k} \psi^{(k)}(1 / x)}{k ! x^{-\alpha} L(x)} \\
& -\frac{(-1)^{d-1} x^{1-d} \psi_{-}^{(d-1)}(1 / x)}{(d-1) ! x^{-\alpha} L(x)} .
\end{aligned}
$$


Now (12) implies that, as $x \rightarrow \infty$, the right-hand side converges to

$$
1-\sum_{k=1}^{d-1} \frac{b_{k}}{k !}=\frac{\Gamma(d-\alpha)}{\Gamma(d) \Gamma(1-\alpha)}>0,
$$

where $\Gamma$ denotes the gamma function. In particular, therefore, $1 / R \in \mathcal{M}\left(\Phi_{\alpha}\right)$ and the proof is complete.

Proof of $(b)$. Suppose first that $1 / R \in \mathcal{M}\left(\Phi_{1}\right)$. Thus, if $G$ denotes the distribution function of $1 / R$ then $\bar{G} \in \mathcal{R}_{-1}$. To show that

$$
L(x)=x\left\{1-\psi\left(\frac{1}{x}\right)\right\}, \quad x \in(0, \infty),
$$

is slowly varying, make a change of variable and use a binomial expansion to write

$$
\begin{aligned}
L(x) & =x\left\{1-\int_{0}^{x}\left(1-\frac{s}{x}\right)^{d-1} \mathrm{~d} G(s)\right\} \\
& =x\left\{1-\sum_{k=0}^{d-1}\left(\begin{array}{c}
d-1 \\
k
\end{array}\right)(-1)^{k} x^{-k} \int_{0}^{x} s^{k} \mathrm{~d} G(s)\right\} \\
& =x \bar{G}(x)+(d-1) \int_{0}^{x} s \mathrm{~d} G(s)-\sum_{k=2}^{d-1}\left(\begin{array}{c}
d-1 \\
k
\end{array}\right)(-1)^{k} x^{-k+1} \int_{0}^{x} s^{k} \mathrm{~d} G(s) .
\end{aligned}
$$

Furthermore, partial integration gives $\int_{0}^{x} s \mathrm{~d} G(s)=-x \bar{G}(x)+\int_{0}^{x} \bar{G}(s) \mathrm{d} s$ and, hence,

$$
L(x)=\{\kappa(x) \lambda(x)+(d-1)\} \int_{0}^{x} \bar{G}(s) \mathrm{d} s, \quad x \in(0, \infty),
$$

where

$$
\kappa(x)=\frac{x \bar{G}(x)}{\int_{0}^{x} \bar{G}(s) \mathrm{d} s}, \quad \lambda(x)=2-d-\sum_{k=2}^{d-1}\left(\begin{array}{c}
d-1 \\
k
\end{array}\right)(-1)^{k} \frac{\int_{0}^{x} s^{k} \mathrm{~d} G(s)}{x^{k} \bar{G}(x)} .
$$

Now, $\kappa(x) \rightarrow 0$ as $x \rightarrow \infty$ by Karamata's theorem, while

$$
\lambda(x) \rightarrow 2-d-\sum_{k=2}^{d-1}\left(\begin{array}{c}
d-1 \\
k
\end{array}\right)(-1)^{k} \frac{1}{k-1}
$$

by Proposition A5.8 of [14]. This means that

$$
\lim _{x \rightarrow \infty} \kappa(x) \lambda(x)+(d-1)=d-1 .
$$

It then follows that $L$ is slowly varying, because $\int_{0}^{x} \bar{G}(s) \mathrm{d} s$ itself has this property in view of Karamata's theorem. In other words, $1-\psi\left(x^{-1}\right) \in \mathcal{R}_{-1}$, as claimed.

The case when $1 / R \in \mathcal{M}\left(\Phi_{\alpha}\right)$ with $\alpha \in(1, \infty)$ is simpler. Then $\mathrm{E}\left(R^{-1-\varepsilon}\right)<\infty$ whenever $\varepsilon$ is such that $1+\varepsilon<\alpha$. Now if $Y$ is a $\operatorname{Beta}(1, d-1)$ random variable which is independent of $R$, it is easily checked that $\operatorname{Pr}(1 / Y>x) \in \mathcal{R}_{-1}$. Breiman's theorem then implies that $\operatorname{Pr}\{1 /(R Y)>x\} \in \mathcal{R}_{-1}$, i.e. $1-\psi\left(x^{-1}\right) \in \mathcal{R}_{-1}$. If $1 / R \in \mathcal{M}(\Lambda)$ or $1 / R \in \mathcal{M}\left(\Psi_{\alpha}\right)$, then all moments of $1 / R$ are finite. In particular, $\mathrm{E}\left(R^{-1-\varepsilon}\right)<\infty$ again holds and we may use the same argument as above to conclude. 


\section{Acknowledgements}

The authors are grateful to Paul Embrechts, Christian Genest, and Alexander J. McNeil for useful comments and several fruitful discussions on this topic. Thanks are due to an anonymous referee for helpful suggestions and for raising the question of whether complete monotonicity guarantees the existence of the lower tail-dependence coefficient. The research of the second author was supported by grants from the Natural Sciences and Engineering Research Council of Canada and the Fonds québécois de la recherche sur la nature et les technologies.

\section{References}

[1] Ballerini, R. (1994). Archimedean copulas, exchangeability, and max-stability. J. Appl. Prob. 31, 383-390.

[2] Barbe, P., Fougères, A.-L. And Genest, C. (2006). On the tail behavior of sums of dependent risks. ASTIN Bull. 36, 361-373.

[3] Bingham, N. H., Goldie, C. M. and Teugels, J. L. (1989). Regular Variation (Encyclopaedia Math. Appl. 27). Cambridge University Press.

[4] Breiman, L. (1965). On some limit theorems similar to the arc-sin law. Teor. Verojat. Primen. 10, 351-360.

[5] CApÉraÀ, P., Fougères, A.-L. ANd Genest, C. (2000). Bivariate distributions with given extreme value attractor. J. Multivariate Anal. 72, 30-49.

[6] Charpentier, A. AND Juri, A. (2004). Limiting dependence structure for credit default. Working paper 2004-16.

[7] Charpentier, A. And Segers, J. (2007). Lower tail dependence for Archimedean copulas: characterizations and pitfalls. Insurance Math. Econom. 40, 525-532.

[8] Charpentier, A. And Segers, J. (2009). Tails of multivariate Archimedean copulas. J. Multivariate Anal. 100, 1521-1537.

[9] Clayton, D. G. (1978). A model for association in bivariate life tables and its application in epidemiological studies of familial tendency in chronic disease incidence. Biometrika 65, 141-151.

[10] Cline, D. B. H. and Samorodnitsky, G. (1994). Subexponentiality of the product of independent random variables. Stoch. Process. Appl. 49, 75-98.

[11] Coles, S., Heffernan, J. and Tawn, J. (2000). Dependence measures for extreme value analyses. Extremes 2 , 339-365.

[12] De HaAn, L. and Ferreira, A. (2006). Extreme Value Theory. Springer, New York.

[13] Demarta, S. (2007). The copula approach to modeling multivariate extreme values. Doctoral Thesis, ETH Zürich.

[14] Embrechts, P., Klüppelberg, C. and Mikosch, T. (1997). Modelling Extremal Events (Appl. Math. 33). Springer, Berlin.

[15] FALK, M. AND ReISs, R.-D. (2005). On Pickands coordinates in arbitrary dimensions. J. Multivariate Anal. 92, 426-453.

[16] Frees, E. W. and Valdez, E. A. (1998). Understanding relationships using copulas. N. Amer. Actuarial J. 2, $1-25$.

[17] Genest, C. And Rivest, L.-P. (1989). A characterization of Gumbel's family of extreme value distributions. Statist. Prob. Lett. 8, 207-211.

[18] Hult, H. And LindSKOG, F. (2002). Multivariate extremes, aggregation and dependence in elliptical distributions. Adv. Appl. Prob. 34, 587-608.

[19] Joe, H. (1993). Multivariate dependence measures and data analysis. Comput. Statist. Data Anal. 16, $279-297$.

[20] JuRI, A. AND WÜthrich, M. V. (2002). Copula convergence theorems for tail events. Insurance Math. Econom. 30, 405-420.

[21] Juri, A. AND WÜthrich, M. V. (2003). Tail dependence from a distributional point of view. Extremes 6, 213-246.

[22] Larsson, M. (2008). Tail properties of multivariate Archimedean copulas. Masters Thesis, ETH Zürich.

[23] Marshall, A. W. and Olkin, I. (1988). Families of multivariate distributions. J. Amer. Statist. Assoc. 83, 834-841.

[24] McNeil, A. J. ANd NeŠLehovÁ, J. (2009). Multivariate Archimedean copulas, $d$-monotone functions and $\ell_{1}$-norm symmetric distributions. Ann. Statist. 37, 3059-3097.

[25] McNeIL, A. J. ANd NeŠLehovÁ, J. (2010). From Archimedean to Liouville copulas. J. Multivariate Anal. 101, 1772-1790.

[26] McNeil, A. J., Frey, R. And Embrechts, P. (2005). Quantitative Risk Management. Princeton University Press.

[27] Nelsen, R. B. (2006). An Introduction to Copulas, 2nd edn. Springer, New York.

[28] OAKes, D. (1994). Multivariate survival distributions. J. Nonparametric Statist. 3, 343-354. 
[29] Resnick, S. I. (1987). Extreme Values, Regular Variation, and Point Processes. Springer, New York.

[30] Resnick, S. I. (2007). Heavy-Tail Phenomena. Springer, New York.

[31] Schönbucher, P. (2003). Credit Derivatives Pricing Models. John Wiley, Chichester.

[32] Schweizer, B. And Sklar, A. (1983). Probabilistic Metric Spaces. North-Holland Publishing, New York.

[33] Zhang, D., Wells, M. T. and Peng, L. (2008). Nonparametric estimation of the dependence function for a multivariate extreme value distribution. J. Multivariate Anal. 99, 577-588. 